Document downloaded from:

http://hdl.handle.net/10251/172311

This paper must be cited as:

López-Maldonado, G.; Pérez Zuriaga, AM.; Moll Montaner, S.; García García, A. (2020). Analysis of Overtaking Maneuvers to Cycling Groups on Two-Lane Rural Roads using Objective and Subjective Risk. Transportation Research Record. 2674(7):148-160. https://doi.org/10.1177/0361198120921169

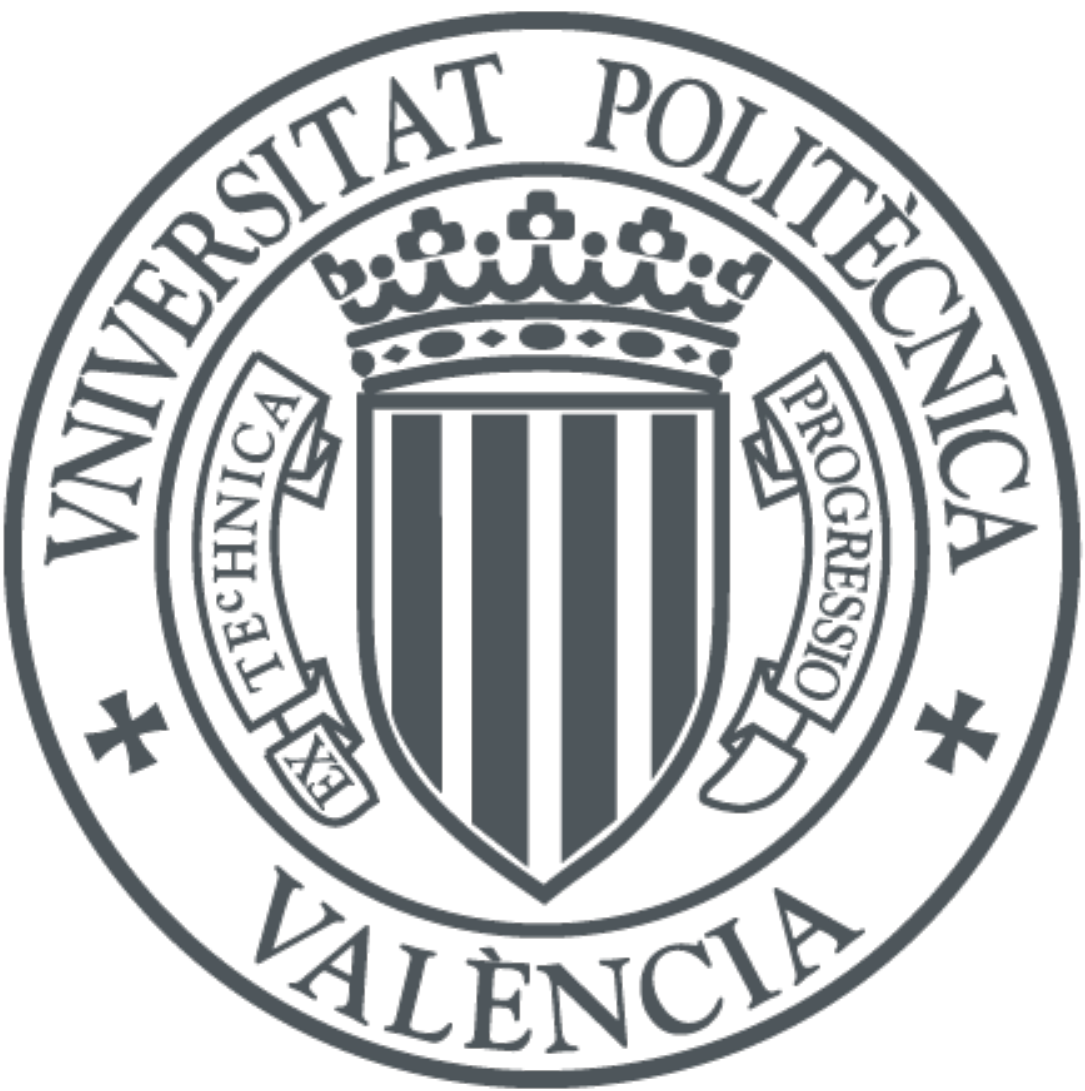

The final publication is available at

https://doi.org/10.1177/0361198120921169

Copyright Transportation Research Board

Additional Information 


\section{Analysis of Overtaking Maneuvers to Cycling Groups on Two-Lane Rural Roads Using Objective and Subjective Risk}

Griselda López*

Assistant Professor

Highway Engineering Research Group (HERG), Universitat Politècnica de València

Email: grilomal@,tra.upv.es

ORCID: 0000-0001-9012-0599

\section{Ana María Pérez-Zuriaga}

Assistant Professor

HERG, Universitat Politècnica de València

Email: anpezu@,tra.upv.es

ORCID: 0000-0002-8434-1106

\section{Sara Moll}

PhD Student

HERG, Universitat Politècnica de València

Email: $\underline{\text { samolmon@upvnet.upv.es }}$

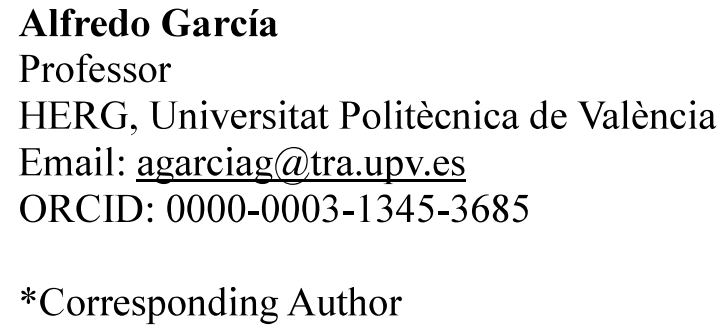

*Corresponding Author

Word count: 6,238 words text +2 tables $\times 250$ words (each) $=6,738$ words

Submission Date: July 30, 2019 


\section{ABSTRACT}

2 In Spain, the presence of cyclists' groups riding on two-lane rural roads in a single file or in parallel 3 line is growing. The number of overtaking maneuvers to them is also increasing. This is one of the most dangerous interaction between motor vehicles and bicycles. However, the risk of these maneuvers has not been deeply analyzed.

This research analyzes the objective and the subjective risk of overtaking maneuvers to cyclists' groups. During this maneuver, the motorized vehicle overtakes the bicycles with a certain speed and lateral distance. These are the surrogate measures used to analyze the objective risk, whereas the subjective risk was analyzed based on the subjective risk perception that 10 cyclists riding instrumented bicycles (in different group configurations) indicated when every motor vehicle overtook them.

Results shown that the cyclists most exposed to the overtaking maneuver are those at the front and at the rear of the group. Regarding to the configuration, the risk is higher in parallel lines, since the lateral clearance is lower compared with a single line. It is even higher when the overtaking maneuver is flying, which is usually performed at higher speeds and lower lateral clearance.

The subjective risk perception increases with higher speed and lower lateral clearance, being higher at the rear positions. Overtaking in which lateral distance is lower than $1.5 \mathrm{~m}$ are perceived as the riskiest. These results provide scientific recommendations to enhance safety for cyclists' groups, and to integrate cycling with vehicular traffic on two-lane rural roads.

Keywords: Cyclists' group, Two-lane rural road, Overtaking bicycle, Risk perception, Road safety 


\section{INTRODUCTION}

In Spain cycling as a sport on two-lane rural roads has increased in the last years, raising new safety concerns. According to (1), there were 8065 fatal-and-injury crashes involving cyclists in 2017, resulting in 78 deaths, 694 serious injuries, and 7075 minor injuries. Although most of these crashes occurred in urban areas (72\%), 63\% of fatalities took place on two-lane rural roads $(90 \%$ of the Spanish road network). Most of the two-lane rural roads in Spain have no cycle lanes, requiring drivers to interact with cyclists, usually by overtaking them.

Compared crashes between urban and rural areas, Boufous et al. (2) indicated that those occurring in rural areas were $28 \%$ more likely to result in severe injury. Cyclists and drivers, on two-lane rural roads share the network. They differ in term of speed, size, weight, and vulnerability (3). In addition, the presence of cyclists on the roads can be considered annoying by drivers (4). Thus, the interaction between cyclists and drivers, especially during overtaking, is a point of interest for researchers.

Overtaking maneuver is considered one of the most dangerous interactions, due to the higher speed of motor vehicles and the lateral clearance necessary to perform the maneuver. The lateral clearance between the cyclist and the vehicle while the vehicle is passing can be used as a surrogate safety measure for safe overtaking (5). Previous research has showed how lateral clearance can be influenced by factors related to the road, the traffic, the vehicle and the cyclist. However, the characterization of drivers-cyclist interaction requires taking also into account the speed of the overtaking vehicle (6). Speed is a significant factor of the aerodynamic forces between overtaken and overtaking vehicles (7); having influence on the severity of rural bicycle crashes (2).

Previous researches explored overtaking maneuvers collecting naturalistic data. The use of these data is the most credited method for understanding road user behavior and improving traffic safety (8). Two data collection methodologies are the most used: instrumented bicycles and driving simulator.

Some studies used instrumented bicycle with different equipment to gather data from overtaking motorists. Most of them focused on lateral clearance between overtaking vehicle and overtaken bicycle on two-lane rural roads. Chapman and Noyce $(9,10)$ investigated the effect of different rural road features on driver's behavior during the overtaking. Their results showed that the presence and the width of the shoulder is a significant factor with positive effect on the lateral clearance distance. Hereinafter, Chapman (11) evaluated the influence of whether a cycle lane exists. As a result, it was observed that drivers do not have a clear idea of what the distance looks like, and they leave more than twice the minimum distance required, whether or not there is a cycle lane. It was also concluded that the cycle lane reduces the invasion of the opposite lane by the overtaking vehicle and keeps its trajectory more constant.

Walker et al. (6) used an instrumented bicycle to investigate whether cyclists' appearance had influence on the lateral distance performed while overtaking, concluding that drivers do not vary their lateral clearance according to the appearance.

Other studies were focused on both lateral clearance and overtaking speed. Dozza et al. (5) identified four overtaking phases and quantified the corresponding driver comfort zones. The effect of factors such as the presence of oncoming vehicles, vehicle speed or maneuver type on driver comfort zone boundaries was investigated. The authors' results showed the need for legislation on minimum overtaking distance stratified by vehicle speed.

The effect of the motor vehicles' speed and the lateral distance during overtaking an individual cyclist, was also investigated by García et al. (12) using an instrumented bicycle. The study was carried out in seven road segments with different lane and shoulder widths. The main findings were that the lateral distance decreases on narrow roads. In addition, the speed is different 
depending on the type of the overtaking maneuvers: flying or accelerative, being accelerative maneuvers those in which drivers slow down and follow the cyclist for some time before passing. Dozza et al. (5) explored both types of maneuvers. They concluded that lateral clearance is larger in accelerative maneuvers possibly because drivers who have larger comfort zone boundaries are also more likely to slow down before overtaking, especially if there is oncoming traffic.

Recently, Farah et al. (13) developed models that predicted overtaking strategy (flying or accelerative) and lateral distance, using data obtained from a driving simulator experiment. It was concluded that the driving speed was a strong predictor of the strategy of overtaking, showing that drivers with higher speeds are more likely to perform a flying maneuver.

Overtaking speed, lateral clearance and/or aerodynamical effect allow one to analyze the objective risk of an overtaking maneuver (14). However, a complete understanding of the phenomenon of overtaking cyclists requires knowing the cyclist risk perception (subjective risk). Up to now, the data have been mainly collected by surveys, being most of them in urban environment $(15,3)$. Garcia et al. (12) and Llorca et al. (14) collected cyclist risk perception data on field. Llorca et al. (14) obtained these data performing an interview on cyclists. They analyzed the relationship between lateral clearance, vehicle speeds and aerodynamic forces and the subjective risk perception. They collected data related to opposing lane occupation, vehicle type, and whether opposing vehicle is present. Results showed that a combined factor of lateral clearance, vehicle type and vehicle speed had a significant correlation with the perceived risk. A research conducted in New Zealand (16) used instrumented bicycles to collect objective and subjective data on overtaking maneuvers considering one cyclist, it was recommended that $1.5 \mathrm{~m}$ distance should be suitable at roads with speeds over $60 \mathrm{~km} / \mathrm{h}$ and $1.0 \mathrm{~m}$ at $60 \mathrm{~km} / \mathrm{h}$ or less.

Most of the studies performed in this field focus only on individual cyclists. However, in Spain, the presence of cyclists' groups on two-lane rural roads is highly common. This fact produces an impact on road facilities, in terms of both road safety and operation. In fact, Spanish traffic regulations (17) states how a group of cyclists should ride throughout the rural road network. Cyclists must perform like a single vehicle when riding in group. To maximize visibility by drivers, cyclists are allowed to ride in parallel, unless low visibility or high traffic conditions exist. Its main objective is not to penalize road operation. However, road safety is not addressed. They indicate only that cyclists must ride as close as possible to the outer edge of the road and motor vehicles overtaking cyclists must keep a minimum lateral distance of $1.5 \mathrm{~m}$. Occupying the opposing lane is permitted even at designated no-passing zones.

There are very few studies that consider cyclists that circulate in group in rural environment. Most of these studies related to cyclists' groups focus on group dynamics in cycling racing environments, and not on road cycling where space is shared with motor vehicles (18). A study on two-lane rural roads considering a group formed by three cyclists was perform by Garcia et al. (19). The overtaking maneuver was studied according to the group configuration, and the objective and the subjective risk perceived by the cyclists in the overtaking maneuvers were analyzed. They concluded that when cyclists ride in parallel the lateral clearance is lower, and that, in general, the cyclists identify less risk when grouping than when they ride alone. They proposed to increase the number of cyclists to study the interaction between vehicles and larger groups. Pérez-Zuriaga et al. (20) studied drivers' behavior when overtaking a 10 cyclists' group riding in different group configurations. They analyzed the relative speed and lateral clearance of the overtaking maneuvers to these groups in different two-lane rural road segments. They concluded that both road features and group configurations have an effect on overtaking speed and lateral clearance.

Debnath et al. (21) examined the factors influencing motor vehicles' compliance with the 
legislated bicycle passing distance rule. It was concluded that non-compliance rates were similar across group or individual riding, but it was a little lower when riding in group in a single file. However, the passing distance was lower for individual riding, whereas cyclists riding two abreast offered the higher passing distance.

The main contribution of this paper, in comparison with previous research, is the study of overtaking maneuver to cyclists riding in group along two-lane rural roads, considering both objective risk and subjective risk perception. The presence of a cyclists' group can influence how driver perform the overtaking strategy, whether it is accelerative or flying, which can affect the objective risk of the maneuver. In addition, different configurations are allowed in the regulations, however the safety of them has not been investigated in depth. Explore these factors is necessary to integrate cycling with vehicular traffic in two-lane rural roads in Spain. In the scope of this research, the analysis of the carriageway width has not been included. The main aim is correlate objective and subjective risk for cyclists. First, the authors need to know how cyclists perceived the risk according with variables that depend of them (grouping configuration and/or grouping position). For further research, the subjective risk perception will be correlated with the specific road characteristics.

\section{OBJECTIVES AND HYPOTHESES}

The aim of this paper is the analysis of the objective risk and the subjective risk perception for overtaking maneuvers performed to cyclists riding in group. To achieve this, the effect of lateral clearance, overtaking vehicle speed, and their combination with the rider's subjective risk perception when cyclists riding on different group configurations (single file and in parallel lines), will be compared.

The hypotheses of this research are:

- Lateral clearance and relative speed between motorized vehicles and cyclists can be used as surrogate measures to study the objective risk of overtaking maneuvers.

- Overtaking lateral clearance depends on the group configuration.

- Subjective risk perception is different depending on the position in which cyclists is riding within the group.

- Lateral clearance and overtaking vehicle speed influence the cyclist's subjective risk perception.

- Overtaking strategy type (flying or accelerative) performed by the drivers depends on the cyclists' group configurations.

- Flying maneuvers are perceived riskier for cyclists in both group configurations.

- Cyclists' perceived risk is different when driver is overtaking in flying or in accelerative manner.

\section{METHODOLOGY AND DATA DESCRIPTION}

A naturalistic study based on instrumented bicycles to collect data for overtaking maneuver and subjective risk perception was performed. A group formed by ten semiprofessional cyclists rode on two-lane rural roads located in the province of Valencia (Spain). The cyclists who participated in the test usually ride on two-lane rural roads and have experience in riding in groups.

Specific road segments with high cyclist demand were previously identified by the research team $(22,23)$. Five of these road segments were chosen to perform the data collection. All of them are situated on flat terrain, being the main differences focus on: the Average Annual Daily Traffic (ranged between 4,733 vehicles/day to 16,310 vehicles/day); the percentage of heavy vehicles (varying from $1.4 \%$ to $6.4 \%$ ), and the width of the carriageway (lane + shoulder), which ranges 
between 3.5 to $5 \mathrm{~m}$.

\section{Data collection}

During data collection, eight of the cyclists rode with their own bicycles equipped with two high definition video cameras to record information about cyclist environment. The forward-facing video camera facilitated the detection of opposing vehicles and the rear-facing high definition video camera observed the overtaking vehicle approach. The video cameras contain a $1 \mathrm{~Hz}$ GPS to obtain the position.

The other two cyclists rode with fully instrumented bicycle (Figure 1a). In addition to the devices installed in the rest of the bicycles, the fully instrumented bicycles were equipped with a laser system. It consisted of 2 laser rangefinders, perpendicular to bicycle axis, one of them in the front part of the bicycle and the other in the rear, being the distance between them known. The sensor provides the relative speed of the overtaking vehicle, after computing the time interval between the measurements of the two rangefinders. It also measures the distance from the bicycle to the overtaking vehicle body and the length of the overtaking vehicle. All these data are stored in a txt file.

\section{[Insert here Figure 1]}

In order to collect the cyclists' perceived risk when motor vehicle is overtaking, specific devices were developed (see Figure 1b). The risk device is connected to a series of LED lights situated at the rear of the bicycle, in the recording area of the rear camera. When cyclist presses a risk value on the device, the corresponding light comes on, which is recorded in the video.

The devices are placed on the handlebars, allowing the cyclist to indicate the perceived risk for every maneuver, with five buttons selector, indicating five risk levels: from 1 (green color is perceived as the safest) to 5 (black color is perceived as the most dangerous). It should be taken into account that this ranking is a subjective and relative classification, as it depends on the cyclist.

Instrumented bicycles were located at the front and at the rear of the group in order to obtain data from the beginning to the end of the overtaking maneuver. This location is highlighted in Figure 1c.

According to Spanish regulations groups can ride in a single file (SF) or in parallel lines (PL). At each road segment, the group performed different rounds following these configurations, as is shown in the Figure 1c. To reduce individual bias in subjective risk, tests were carried out for several cyclists in each position. Members of the research team were located at the end and at the beginning of the road segments to verify the tests. Data collection were carried out during weekend morning (Saturday and Sunday), since it is when more groups ride and drivers expect to find them. All tests took place under good weather conditions and dry pavement.

\section{Data reduction}

In order to obtain the variables under study, a pretreatment procedure for data was needed. Data recording came from two sources: video cameras and laser speedometers. A synchronization of video, laser and risk device data is performed to obtain a complete database.

According to the criteria stablished by García et al. (12), the following overtaking dynamic variables were characterized:

- Relative Vehicle Speed (Vr): relative speed between the overtaking vehicle and the bicycle/s.

- Bicycle speed (Vb).

- Vehicle Speed (Speed): Vr + Vb. 
- Lateral Spacing (Ls): distance between the center of the bicycle and the body of the motor vehicle collected by the laser.

- Lateral Clearance (Lat_Clearance):

- Lat_Clearance for heavy vehicles: Ls- half handlebar width $(0.15 \mathrm{~m})$

- Lat_Clearance for other vehicles: Ls - half handlebar width $(0.15 \mathrm{~m})$ - vehicle side mirror width $(0.12 \mathrm{~m})$.

All these variables are obtained at the beginning (Beg) and at the end (End) position of the overtaking maneuver. Variables remarked in bold were used in this study. In addition, with the visualization of the videos, other variables were also registered:

- The overtaking maneuver type (flying vs. accelerative), being flying defined as the maneuver in which the overtaking vehicle does not accelerate during the maneuver keeping the same speed; and accelerative as the maneuver in which the overtaking vehicle reduces its speed and follows the cyclist before overtaking.

- The opposite lane occupation (total, partial, no occupation), being total when cars overtake using the opposite lane during the entire maneuver; partial if cars use both lanes to overtakes (at the begin or at the end); or no when cars do not use the opposite lane to perform the maneuver. In Spain, cyclist can be overtaken even when center line is continuous (no-passing zone).

- Subjective risk perception registered for the ten cyclists (from P1 to P10) with 5 possible values for every maneuver (1 - very low risk perception; 2- low risk perception; 3 - medium risk perception; 4 - high risk perception; 5 - very high risk perception).

After data preprocessing, a total of 1,608 overtaking maneuvers were registered. From these data, 361 interactions have been removed (due to malfunctions of the equipment or overtaking at intersections), leaving a total of 1,292 valid maneuvers. From this data, 620 overtakings in which group is riding with the habitual configuration used by cyclists (SF and PL) will be analyzed.

\section{RESULTS AND DISCUSSION}

\section{Descriptive analysis}

According to the analyzed group configuration, there are 309 overtakes to cyclist riding in a single file (SF), and the remainder 311 are to parallel lines (PL). The vehicle types involved in the maneuvers was mainly passenger cars (about $87 \%$ of the registered maneuvers), followed by motorcycles $(7 \%)$, large vans $(4 \%)$ and heavy vehicles $(1 \%)$. The main characteristics of the overtaking maneuvers are presented in Table 1.

The results show that most overtaking maneuvers registered are flying $(87 \%)$, and only $13 \%$ are accelerative overtaking maneuvers. Observing the results by configurations, when the cyclists ride in parallel, the highest percentage of accelerative maneuvers occurs (19\%).

Spanish regulations allow the opposing lane occupation even at designated no-passing zones to perform the overtaking maneuvers to cyclists. This fact is correlated with the percentages of opposing lane occupation obtained, being partial in most maneuvers (64\% in SF vs. $70 \%$ in PL). The occupation of the opposite lane generates a risk situation of frontal collision with the oncoming traffic. At the same time, the non-occupation of the opposite lane can generate not enough lateral clearances between vehicle and bicycles. 
López, Pérez-Zuriaga, Moll-Montaner and García

1 TABLE 1. Description of the overtaking maneuvers characteristics.

\begin{tabular}{|c|c|c|c|c|}
\hline \multirow{2}{*}{ Variables } & \multicolumn{2}{|c|}{ SF } & \multicolumn{2}{|c|}{ PL } \\
\hline & N (\%) & Mean (SD) & N (\%) & Mean (SD) \\
\hline $\begin{array}{l}\text { Overtaking Maneuver Type } \\
\text { Flying } \\
\text { Accelerative }\end{array}$ & $\begin{array}{l}284(92 \%) \\
25(8 \%)\end{array}$ & & $\begin{array}{l}253(81 \%) \\
58(19 \%)\end{array}$ & \\
\hline $\begin{array}{l}\text { Opposite lane occupation at the begin } \\
\text { Total } \\
\text { Partial } \\
\text { No occupation }\end{array}$ & $\begin{array}{l}35(11 \%) \\
198(64 \%) \\
76(25 \%)\end{array}$ & & $\begin{array}{l}77(25 \%) \\
219(70 \%) \\
15(5 \%)\end{array}$ & \\
\hline $\begin{array}{l}\text { Opposite lane occupation at the end } \\
\text { Total } \\
\text { Partial } \\
\text { No occupation }\end{array}$ & $\begin{array}{l}29(9 \%) \\
169(55 \%) \\
108(35 \%)\end{array}$ & & $\begin{array}{l}67(22 \%) \\
188(61 \%) \\
53(17 \%)\end{array}$ & \\
\hline $\begin{array}{l}\text { Speed }(\mathrm{km} / \mathrm{h}) \\
\text { Speed_Beg }(\mathrm{km} / \mathrm{h}) \\
\text { Speed_End }\end{array}$ & & $\begin{array}{l}67.03(17.28) \\
64.96(11.36) \\
\end{array}$ & & $\begin{array}{l}63.31(16,69) \\
63.31(10,81) \\
\end{array}$ \\
\hline $\begin{array}{l}\text { Lateral clearance }(\mathrm{m}) \\
\text { Lat_Clearance_Beg } \\
\text { Lat_clearance_End }\end{array}$ & & $\begin{array}{l}2.08(0.55) \\
2.44(0.53)\end{array}$ & & $\begin{array}{l}1.92(0.55) \\
2.33(0.60)\end{array}$ \\
\hline
\end{tabular}

The occupation of the opposite lane is higher when cyclists ride in parallel. In addition, comparing with SF, when cyclists ride in parallel, most maneuvers are accelerative (19\% vs. $8 \%$ ). According with Farah et al. (13), the accelerative maneuver requires a previous following to the cyclists that allows to wait for a better visibility to overtake and, therefore, these maneuvers are safer than flying.

The values for the vehicle speed and the lateral clearance between the overtaking vehicle and the bicycles at the beginning and at the end of the overtaking maneuver, for each group configuration, were studied (see Table 1). Overtaking speeds are very similar in both configurations. However, in the SF configuration they are slightly higher. Regarding lateral clearance, in a SF configuration they are higher. Then, a higher opposite lane occupation (in PL is higher than in SF) does not imply a higher lateral clearance in the overtaking. Garcia et al. (19) carried out a study with a group of three cyclists in the same region of Spain, obtaining the lower lateral separations when the cyclists circulated in parallel.

In general, at the beginning of the overtaking maneuver, overtaking vehicle speeds are higher and lateral clearance are lower. These results are according with Pérez-Zuriaga et al. (20) where more group configurations were analyzed.

\section{Subjective risk perception}

In this section, the results on how cyclists perceived the maneuvers when riding at SF or PL are presented. Figure 2 shows the percentages of the subjective risk perception level (pressed by each cyclist, in each overtaking maneuver), in the ten group positions, being P1 front and P9/P10 rear (see Figure 1c). In addition, the average of the risk level in the different positions is also presented. These values are shown for both analyzed group configurations, SF and PL. 
As it is shown in Figure 2, the positions in which cyclists perceive the highest level of risk are mainly, the front and rear. In the SF configurations most cyclists feel a very low or low risk in the most maneuvers. However, positions P10 and P9 are the positions in which the subjective level perception is higher, followed by $\mathrm{P} 1$.

In PL configuration, the risk perception values are distributed more homogeneously among the different levels. The position remarked in grey color are those where cyclists are riding closer to motorized traffic (odd position). In general, these positions present higher perceived risk compared with the other positions in which cyclists are not in contact with traffic. In addition, in PL configuration the position with the higher subjective risk levels are P7, P9 and P10, respectively, located at the rear position in the overtaking maneuver; and P1, P2 and P3 located at front position.

According to these results, a subjective risk perception for rear (SRisk_rear) and front position (SRisk_front) of the group was calculated depending on the configuration, following this criterion:

- SRisk_front_SF= max subjective risk level (P1 and P2); SRisk_front_PL = max subjective risk level (P1, P2 and $\mathrm{P} 3)$.

- SRisk rear_SF= max subjective risk level (P10 and P9); SRisk_rear_PL = max subjective risk level (P10, $\mathrm{P} 9$ and $\mathrm{P} 7)$.

\section{[Insert here Figure 2]}

\section{Type of overtaking maneuvers vs. subjective risk perception}

Motorized vehicles can overtake a bicycle group through two overtaking strategies: flying or accelerative. As it was previously shown in Table 1, most of the overtaking maneuver recorded in this study are flying (87\%). The mean and standard deviation of the speed and lateral clearance per overtaking strategy (accelerative vs. flying) for each group configuration, taking into account data from the beginning and from the end, of the overtaking maneuver analyzed are presented in Table 2.

TABLE 2. Descriptive statistics of speed and lateral clearance in flying vs. accelerative overtaking maneuver

\begin{tabular}{l|l|l|l|l}
\hline \multirow{2}{*}{ Variables } & \multicolumn{2}{|c|}{ SF } & \multicolumn{2}{c}{ PL } \\
\cline { 2 - 5 } & $\begin{array}{l}\text { Accelerative } \\
\text { Mean (SD) }\end{array}$ & $\begin{array}{l}\text { Flying } \\
\text { Mean (SD) }\end{array}$ & $\begin{array}{l}\text { Accelerative } \\
\text { Mean (SD) }\end{array}$ & $\begin{array}{l}\text { Flying } \\
\text { Mean (SD) }\end{array}$ \\
\hline $\begin{array}{l}\text { Speed (km/h) } \\
\text { Speed_Beg (km/h) }\end{array}$ & $50.68(9.52)$ & $68.36(17.56)$ & $50.60(7.87)$ & $66.17(16.83)$ \\
Speed_End & $54.83(6.86)$ & $65.99(11.24)$ & $54.52(6.66)$ & $65.93(10.43)$ \\
\hline Lateral clearance (m) & & & & \\
Lat_Clearance_Beg & $2.19(0.58)$ & $2.08(0.54)$ & $1.89(0.53)$ & $1.93(0.55)$ \\
Lat_Clearance_End & $2.40(0.44)$ & $2.16(0.53)$ & $2.26(0.43)$ & $2.00(0.63)$ \\
\hline
\end{tabular}

As it is shown in Table 2, in both configurations (SF and PL) flying maneuver present higher speeds than accelerative maneuver in both positions. The mean lateral clearance for SF configuration is higher for accelerative maneuver. In contrast, in PL configuration, flying and accelerative maneuver present practically the same mean lateral clearance at the beginning of the maneuver. These results agree with those obtained by Farah et al. (13) where the mean lateral clearance and the mean overtaking speed were higher for flying overtaking maneuver.

Lateral clearance was larger in accelerative maneuver possibly because drivers who had larger comfort zone boundaries were also more likely to slow down before overtaking, especially 
if there was oncoming traffic (5). Drivers performing flying maneuver approached and passed the cyclist faster because their relative speed were higher than those performing accelerative maneuver (13).

In order to know which configuration is safer, the overtaking strategy performed by drivers, according to the subjective risk perception, was also analyzed. Figure 3 shows that, in general, flying overtaking maneuver present higher levels of subjective risk perception than accelerative overtaking maneuver, especially at the beginning of the overtaking. These results are in concordance with Dozza el al. (5) and Farah et al. (13), who indicated that accelerative overtaking maneuvers are safer than flying overtaking maneuvers, since in this type of maneuver vehicles circulate at lower speeds, control the interaction with the oncoming traffic and leave larger clearances to the cyclists.

\section{[Insert here Figure 3]}

Regarding group configurations, PL presents higher level of subjective risk perception, in the both positions analyzed (beginning and end), remark that in these positions lateral clearance presents differences statistically significant between SF and PL. The results of subjective risk perception of the cyclists are contrary to the cyclists' preferences analyzed by López et al. (24). In this study, a cyclist's survey carried out in Spain, cyclists indicated a preference for riding in parallel, because they felt safer.

\section{Subjective risk perception vs. Objective risk}

The objective risk of an overtaking maneuver can be evaluated using surrogate measures such as lateral clearance, speed or a combination of both. The first aim is comparing the objective risk with the subjective risk perception, at the beginning and at the end of group positions, being these positions the critical point of the overtaking maneuvers. In addition, these results are compared among the different group configurations.

Figure 4 shows the box plot of the variables speed and lateral clearance by subjective risk level (for the first and the last cyclists) in the SF and PL configurations. The differences on the median and distribution of these variables (speed and lateral clearance) among different levels of subjective risk perceptions are shown. The factor plotted in the vertical axis is the subjective risk perception (ranking from 1 to 5), and the dependent variables are speed or lateral clearance. Differences among the medians were checked by using the nonparametric Kruskal Wallis (K-W) test, as the variables did not come from a normal distribution, at the $95 \%$ confidence level. $\mathrm{K}-\mathrm{W}$ test $p$-values under 0.001 showed differences among the medians.

Regarding speed variable, only at rear position in SF configuration results were significant $\left(F_{S F_{-} \text {Rear }}=3.08, p=0.0167\right)$, the remainder tests were not significant $\left(F_{S F_{-} \text {Front }}=1.57, p=\right.$ $\left.0.2011 ; F_{P L_{\text {FFront }}}=0.30, p=0.8238 ; F_{P L_{-} \text {Rear }}=0.13, p=0.9419\right)$. In fact, the results did not show any clear tendency. As can be seen in Figure 4, regarding with SF configuration, overtaking speeds are, in general, higher at rear position, this fact can be correlated with higher subjective risk perception indicated by cyclists in these positions. In PL, speeds are very similar in both positions of the group.

The average speed between 1,2 and 3 risk levels are very similar at the front and at the rear position for both configurations. The results of the level 4 are not representative having a wide dispersion due the sample size (less than 10 values).

Regarding with the lateral clearance, results of the tests were statistically significant $\left(F_{S F_{-} \text {Rear }}=12.74, p<0.001 ; F_{S F_{-} \text {Front }}=15.75, p<0.001 ; F_{P L_{-} \text {Rear }}=11.76, p<\right.$ 
$\left.0.001 ; F_{P L_{-} \text {Front }}=16.83, p<0.001\right)$. As it is showed in Figure 4, there is a clear relation with the subjective risk perceived: in both configurations (PL and SF) risk level perception increases when lateral clearance decreases. Overtaking in which lateral distance is lower than the minimum legal $(1.5 \mathrm{~m})$ are perceived as the riskiest (more than level 3 ).

\section{[Insert here Figure 4]}

\section{Correlation between subjective risk perception and objective risk}

A correlation analysis was conducted to assess the effect of the objective risk variables on the subjective risk perception, by performing Pearson test. Different surrogate measures were used to evaluate objective risk, such as overtaking speed, lateral clearance and different variables which combined effects of speed and lateral clearance. The correlation at the rear and front position was evaluated using the following variables: $Y_{1}=$ Speed, $Y_{2}=$ Lat_Clearance, $Y_{3}=$ Speed/Lat_Clearance, $Y_{4}=(\text { Speed })^{2} /$ Lat_Clearance and $Y_{5}=$ variable based on the aerodynamical forces. According with Llorca et al. (14) the variable based on the aerodynamical forces most related to subjective perceiver risk is defined by Equation 1.

$Y_{5}\left(\frac{m^{5}}{s^{2}}\right)=V\left(\frac{m}{s}\right)^{2} *(3-L s(m)) *$ Front area $\left(m^{2}\right)$

Where, values for front area are assigned according with the vehicle category: $2 \mathrm{~m}^{2}$ (Motorcycles and Passenger cars), $3.7 \mathrm{~m}^{2}$ (Large Van) and $6 \mathrm{~m}^{2}$ (Heavy vehicles and Bus). These values were taken according to the recommendations of the Queensland Government Department of Transport and Main Roads (25).

Results showed that in both positions (rear and front), only the speed $\left(Y_{l}\right)$ was not correlated with subjective risk, neither front $(\mathrm{p}=0.2112)$ and rear $(\mathrm{p}=0.1458)$ positions. The variables most correlated in both positions were $V_{3}$ followed by $Y_{5}$. At the front position, the correlations were higher, with a moderate positive correlation with $Y_{3}\left(\mathrm{r}_{\mathrm{s}}=0.6052, \mathrm{p}<0.001\right)$ and $Y_{5}$ $\left(\mathrm{r}_{\mathrm{s}}=0.5683, \mathrm{p}<0.001\right)$.

To explore if there are significant differences on $Y_{3}$ variable among the subjective risk perception levels, ANOVA analysis was performed (at the begin and at the end of the overtaking maneuver, for SF and PL configurations). The results indicated that there is a statistically significant difference between the mean of $Y_{3}$ among levels of perceived risk, with a level of $5 \%$ significance $\left(F_{S F_{-} \text {Rear }}=28.20, p<0.001 ; F_{S F_{-} \text {Front }}=27.93, p<0.001 ; F_{P L_{-} \text {Rear }}=12.41\right.$, $\left.p<0.001 ; F_{P L_{-} \text {Front }}=28.64, p<0.001\right)$.

To visualize the levels which present differences with the most objective correlated variable $\left(Y_{3}\right)$, Fisher's LSD-intervals are plotted in Figure 5. One graphic is represented for each group configuration, in the vertical axis is represented the dependent variable $\left(Y_{3}\right)$, and in the horizontal axis is plotted the subjective risk perception.

The analysis of the combined effect of the overtaking speed and lateral clearance shows a general tendency in which when the ratio increases (thus is higher speed and minor lateral clearance), the subjective risk perception increases (see Figure 5). In the SF and PL configuration at the front position there were the same differences between levels: levels 1 and 2 present overlapping, and they have differences statistically significant with levels 3 and 4 . At the rear position in SF there were differences between all the risk levels (the same tendency observed with lateral clearance variable).

Figure 5 shows that the risk perception shows significant differences between all risk levels in both positions and in both configurations analyzed. In addition, the subjective risk perception is higher when speed increases and lateral clearance decreases at front and rear for 
López, Pérez-Zuriaga, Moll-Montaner and García

group positioning in SF and PL.

\section{[Insert here Figure 5]}

\section{CONCLUSIONS}

The aim of this research was to analyze the subjective risk perceived by cyclists during the overtaking maneuvers. In addition, the subjective risk was correlated with the objective risk, defined by surrogate measures registered during the overtaking (lateral clearance, overtaking vehicle speed), for each overtaking maneuver to a cyclist group. It was also analyzed how the maneuver driver strategy is perceived by the group of cyclists.

In order to collect data, a seminaturalistic study with instrumented bicycles was performed. A group of ten cyclists rode in five two-lane rural road segments in the region of Valencia. This group rode in two different configurations: single file (SF) and parallel lines (PL). Ten bicycles were instrumented by two video cameras and a device with five buttons where the cyclists could press the perceived risk for each overtaking maneuver. The front and the rear bicycles of the group were also instrumented with a laser device to collect overtaking speeds and lateral clearances when the overtaking begins and ends.

Results about the occupation of the opposite lane shown that, in general, there is more lane occupation at the beginning of the maneuver. When cyclists rode in parallel more overtaking maneuvers are carried out with opposite lane occupation; however, this does not imply a higher lateral clearance in the overtaking maneuver (this also depends on the lane characteristics). In fact, in a SF configuration, the lateral clearance is higher than in PL. On the other hand, the occupation of the opposite direction increases risk exposure to drivers (the risk of frontal collision for the overtaking vehicles); but also, in most cases (depending on the road characteristics), represents a greater lateral separation between the overtaking vehicles and the bicycle group.

Subjective risk perception of cyclists has been represented for each position inside the group for both studied configurations. The highest perceptions were registered at the front and the rear of the group, being the cyclists that circulate at the rear those who have the highest perception of risk, since they are located in the beginning of the overtaking (where the highest speeds and the lower lateral clearances were recorded). When the group circulates in parallel, the cyclists positioned in the area closest to the passing vehicles register higher risk perceptions.

As expected, the higher the lateral clearance and the lower the speed, imply the safer the rider perception. This agrees with results obtained by Llorca et al. (14) who analyzed overtaking maneuvers to an individual cyclist.

Relation between the perceived risk by cyclists and the overtaking maneuver has also been studied. Results shown that the $87 \%$ of the overtaking maneuver registered were flying maneuver. When the cyclist group rode in PL configuration, there were the major percentage of accelerative maneuver (19\%). This is because, in parallel, cyclists occupy more space in the road and force drivers to follow them before overtaking, especially on narrow roads. Flying overtaking maneuvers present higher levels of subjective risk perception, especially at the beginning of the overtaking. The configuration that presents the lowest subjective risk is, in general, SF.

The combined effect between the overtaking speed and lateral clearance, evaluated as the ratio between these variables, was most correlated variable with the subjective risk perception. In addition, ANOVA results shown that the ratio presents statistically independent values for each level of perceived subjective risk. Then, it can be used as a surrogate measure of the overtaking risk. Using this variable, as a further research line, the identification of safe overtaking according to the width of road will be performed. This result is in the line with the obtained by Llorca et al. (14) who performed a similar test, two-lane rural roads located in the Valencia region, using one 
cyclist. They indicated that the lateral clearance is not the only factor with influenced on the riders' risk perception.

Based on the correlation analysis performed between objective and subjective risk, results show that the cyclists' feeling is rational, and is consistent with the objective risk. The most correlated variable with the subjective risk perception was the ratio between overtaking speed and lateral clearance. The actions from public administrations and road engineers should be based on this measure, being able to act on both risks.

This research helps to better understand how the overtaking maneuvers performed by drivers are perceived by cyclists, considering single file and two abreast group configurations. The findings may inform policy makers on how to successfully, and equitably, integrate cycling with vehicular traffic on two-lane rural roads in Spain. The results of this research could be the basis to advice the cyclists how to ride in a specific configuration when riding in some traffic flow conditions or roads. However, before making these recommendations, further research considering cross-section and different traffic conditions should be carried out. Possible measures can be oriented into speed management on the road, or recommendation for a specific group configuration during periods in which more motorized vehicles are driven depending on road characteristics.

As a limitation of this research it can be highlighted that it is based on surrogate measures, and they would require a validation with crash data, if they were available. In addition, the results of the subjective risk perception are based on ten cyclists, and although the research team try to reduce the individual bias varying the cyclist's position within the group on the different tests, a higher number of cyclists should be considered. As a further research, a more extensive study about subjective risk perception is needed, trying to identify which are the most significant risk factors for cyclist, including overtaking time, different length of groups, and the influence of the road sections for the different group configurations.

\section{AKNOWLEDGMENTS}

The study presented in this paper is part of the research project titled "Road safety countermeasures for two-lane rural roads with group of cyclists (Safe4Bikes)" (SPIP2017-02280), subsidized by the Dirección General de Tráfico (General Directorate of Traffic) of Spain.

\section{AUTHORS CONTRIBUTION}

The authors confirm contribution to the paper as follows: study conception and design: G. López, A.M. Pérez-Zuriaga, S. Moll, A. García; data collection: S. Moll, G. López, A.M. PérezZuriaga; analysis and interpretation of results: G. López, S. Moll, A.M. Pérez-Zuriaga A. García; draft manuscript preparation: G. López, A.M. Pérez-Zuriaga, S. Moll, A. García. All authors reviewed the results and approved the final version of the manuscript.

\section{REFERENCES}

1. Dirección General de Tráfico. Las principales cifras de la Siniestralidad Vial: España 2017. Dirección General de Tráfico, 2018. (ISSN: 2445-0219).

2. Boufous, S., L. de Rome, T. Senserrick, and R. Ivers. Risk factors for severe injury in cyclists involved in traffic crashes in Victoria, Australia. Accident Analysis and Prevention, 2012. 49: 404-409.

3. Chaurand, N., and P. Delhomme. Cyclists and drivers in road interactions: A comparison of 
López, Pérez-Zuriaga, Moll-Montaner and García

perceived crash risk. Accident Analysis and Prevention, 2013. 50: 1176- 1184.

4. Basford, L., S. Reid, T. Lester, J. Thomson and A. Tolmie. Drivers' perceptions of cyclists. TRL Report TRL549. Charging and Local Transport Division, Department for Transport, 2002.

5. Dozza, M., R. Schindler, G. Bianchi-Piccinini, and J. Karlsson. How do drivers overtake cyclists? Accident, Analysis and Prevention, 2016. 88: 29-36.

6. Walker, I., I. Garrard, and F. Jowitt. The influence of a bicycle commuter's appearance on drivers' overtaking proximities: an on-road test of bicyclist stereotypes, high-visibility clothing and safety aids in the United Kingdom. Accident Analysis and Prevention, 2014. 64: 69-77.

7. Ata, M.K., and R.G. Langlois. Factoring cycling in transportation infrastructure: design considerations based on risk exposure. ITE Journal, 2011. 49-53.

8. Dozza. M., and J. Werneke. Introducing naturalistic cycling data: What factors influence bicyclists' safety in the real world? Transportation Research Part F: Traffic Psychology and Behaviour, 2014. 24: 83-91.

9. Chapman, J., and D. Noyce. Observations of driver behavior during overtaking of bicycles on rural roads. Transportation Research Record: Journal of Transportation Research Board, 2012. 2321: 38-45. http://dx.doi.org/10.3141/2321-06.

10. Chapman, J., and D. Noyce. Influence of roadway geometric elements on driver behavior when overtaking bicycles on rural roads. Journal of Traffic and Transportation Engineering, 2014. 1: 28-38.

11. Chapman, J. Evaluation of Lateral Clearance Distances between Vehicles and Bicycles During Overtaking Maneuvers on Rural Roads. Transportation Research Board 95th Annual Meeting, Washington D.C., 2016.

12. García, A., A. Angel-Domenech, C. Llorca, F. Agustín-Gómez, and V. Ferrer. Effects of road geometry on the interaction between bicyclists and motor vehicles on two-way rural highways. 5 th International Symposium on Highway Geometric Design, Vancouver, Canada, 2015.

13. Farah, H., G. Bianchi Piccinini, M. Itoh, and M. Dozza. Modelling overtaking strategy and lateral distance in car-to-cyclist overtaking on rural roads: A driving simulator experiment. Transportation Research Part F: Traffic Psychology and Behaviour, 2019. 63: 226-239. https://doi.org/10.1016/j.trf.2019.04.026

14. Llorca, C., A. Angel-Domenech, F. Agustin-Gomez, and A. Garcia. Motor vehicles overtaking cyclists on two-lane rural roads: Analysis on speed and lateral clearance. Safety Science, 2017. 92:302-310.

15. Winters, M., G. Davidson, D. Kao, and K. Teschke. Motivators and deterrents of bicycling: comparing influences on decision to ride. Transportation, 2011. 38: 153-168. http://dx.doi.org/10.1007/s11116-010-9284-y. 
López, Pérez-Zuriaga, Moll-Montaner and García

16. NZ Transport Agency. Investigating the feasibility of trialling a Minimum Overtaking Gap law for motorists overtaking cyclists in New Zealand. 2016.

17. Ministerio del Interior. Real Decreto 1428/2003, de 21 de noviembre, por el que se aprueba el Reglamento General de Circulación. 2003.

18. Trenchard, H., A. Richardson, E. Ratamero, and M. Perc. Collective behavior and the identification of phases in bicycle pelotons. Physica A: Statistical Mechanics and its Applications, 2014. 405, 92-103.

19. García. A., C. Llorca, and J. Serra-Planelles. Influence of peloton configuration on the interaction between sport cyclists and motor vehicles on two-lane rural roads. Journal of Transportation Safety \& Security, 2020. doi: 10.1080/19439962.2019.1591557.

20. Pérez-Zuriaga, A. M., G. López-Maldonado, S. Moll-Montaner, and A. García. Drivers' behavior overtaking a bicycle peloton on two-lane rural roads. International Cycling Safety Conference. Barcelona, Spain, 2018.

21. Debnath, A. K., N. Haworth, A. Schramm, K. C. Heesch, and K. Somoray. Factors influencing noncompliance with bicycle passing distance laws. Accident Analysis and Prevention, 2018. 115, 137-142.

22. Camacho-Torregrosa, F.J., G. López-Maldonado, S. Moll-Montaner and A. M. Pérez-Zuriaga, D. Llopis-Castelló, and M. Lowry. Identification of cyclist volume patterns in Spain using observations and Strava data. $7^{\text {th }}$ International Cycling Safety Conference, Barcelona, 2018.

23. López, G., F.J. Camacho-Torregrosa, S. Moll-Montaner, A. García. Estimación de la demanda ciclista en carretera usando datos de la plataforma Strava. XIII Congreso de Ingeniería del Transporte, Gijón (Spain), 2018.

24. López, G., I. Martínez, F.J. Camacho-Torregrosa, and A. García. Analysis of cyclist perception and behavior on two-lane rural roads through an online survey. Transportation Research Board 97th Annual Meeting, Washington D.C., 2019.

25. Queensland Government Department of Transport and Main Roads. Queensland Road Rules for Cyclists, 2013. 
List of Figures:

Figure 1. Measurement equipment and cyclists' configurations.

Figure 2. Subjective risk perception by configuration, from 1 (very safe) to 5 (very risky). Figure 3. Analysis of the subjective risk perception of group configuration vs. type of maneuvers.

Figure 4. Box plot of the overtaking speed and lateral clearance among subjective risk perception levels.

9 Figure 5. Means and $95 \%$ LSD intervals for the ratio of overtaking speed and lateral 10 clearance among the different risk perception levels. 


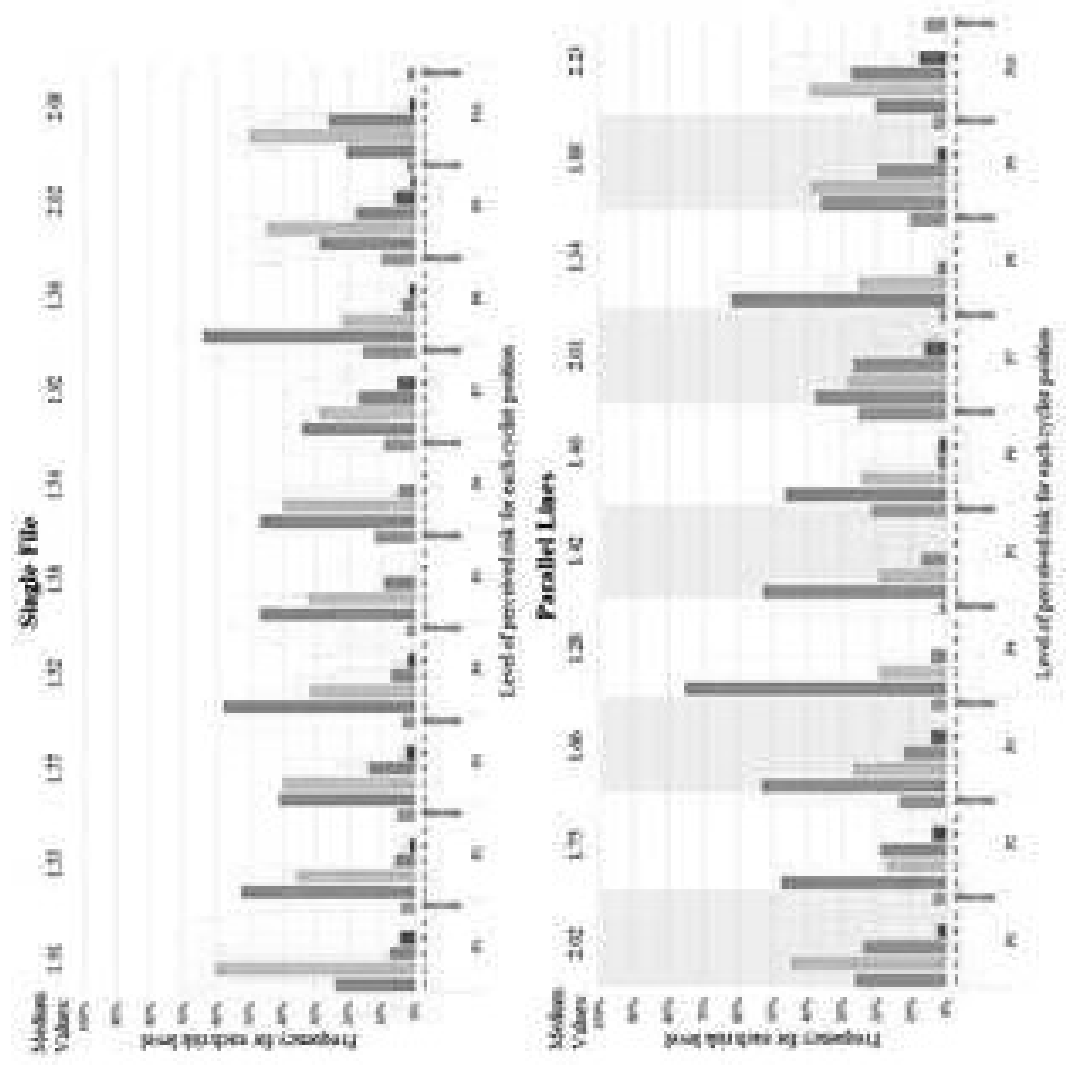

은 

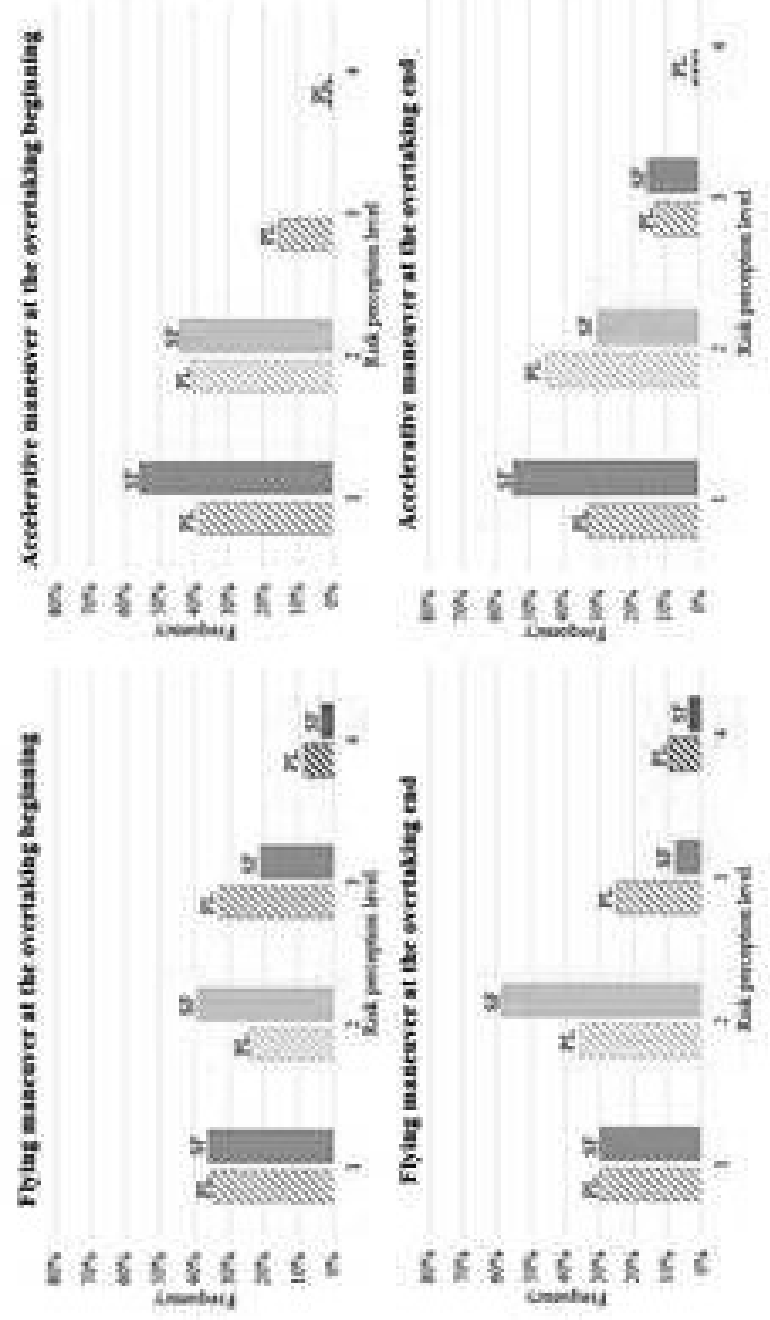

논 


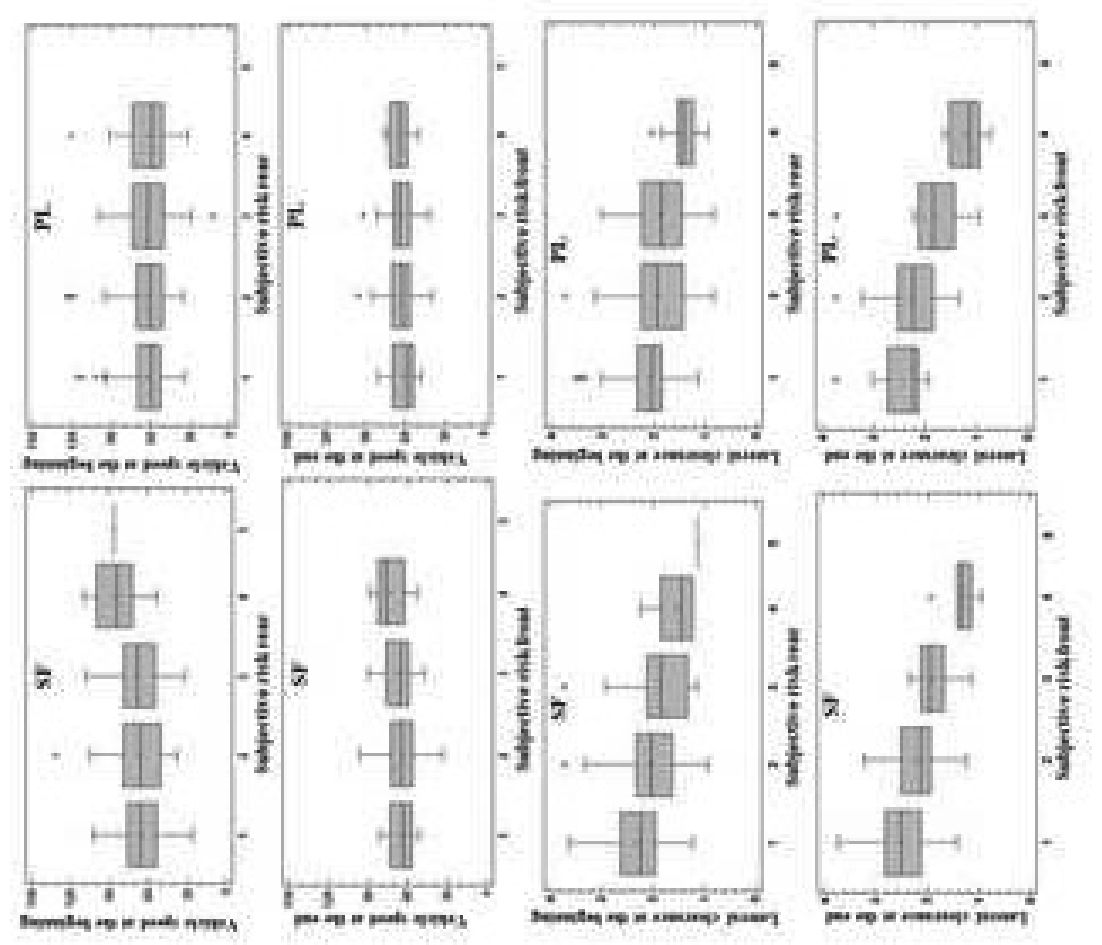

高 

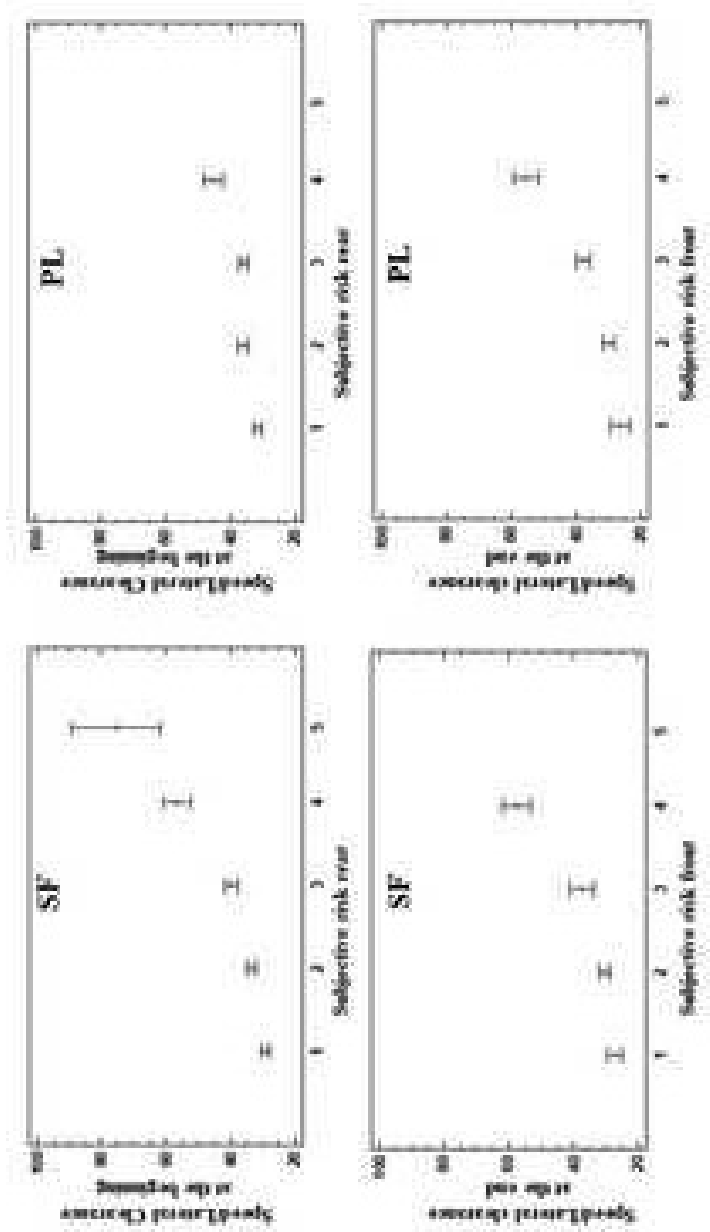

을 